\title{
ON CERTAIN ELEMENTARY TRILINEAR OPERATORS
}

\author{
Michael Christ
}

\section{Introduction}

Consider the family of multilinear operators, acting on locally integrable functions $f_{j}$ defined in $\mathbb{R}^{d}$ by

$$
T\left(f_{1}, \ldots, f_{m}\right)(x)=\int_{|t| \leq 1} \prod_{j=1}^{m} f_{j}\left(S_{j}(x, t)\right) d t,
$$

where the $S_{j}: \mathbb{R}^{d+d} \mapsto \mathbb{R}^{d}$ are surjective linear mappings. In this paper we focus primarily on the trilinear case $m=3$. Writing $S_{0}(x, t) \equiv x$, we exclude degenerate cases by assuming always that for any two indices $i \neq j$, the mapping $(x, t) \mapsto\left(S_{i}(x, t), S_{j}(x, t)\right)$ is invertible; in that case we say that $\left\{S_{j}: 0 \leq j \leq 3\right\}$ is nondegenerate.

Under these hypotheses, for $m=3, T(F)$ is well-defined as a measurable function of $x \in \mathbb{R}^{d}$, for all $F=\left(f_{1}, f_{2}, f_{3}\right)$ belonging locally to $L^{p} \otimes L^{p} \otimes L^{p}$, provided that $p \geq 3 / 2$. Indeed, it follows directly from Fubini's theorem that $T$ maps $L^{1} \otimes L^{1} \otimes L^{\infty}$ to $L^{1}$, and $L^{1} \otimes L^{\infty} \otimes L^{\infty}$ to $L^{\infty}$, and that the same holds if the roles of the functions $f_{j}$ are interchanged in an arbitrary way. Multilinear interpolation then yields the conclusion that $T$ maps $L^{p} \otimes L^{p} \otimes L^{p}$ to $L^{q}$, whenever $1+q^{-1}=3 p^{-1}$ and $3 / 2 \leq p \leq 3$.

Our main result specifies which trilinear expressions (1) are well defined on $L^{p} \otimes L^{p} \otimes L^{p}$ for some $p<3 / 2$; this turns out to be equivalent to the extension of $L^{p} \otimes L^{p} \otimes L^{p} \mapsto L^{q}$ inequalities below $p=3 / 2$. In this range, $T(F)$ will fail to be locally integrable for some $F$, and the natural conclusion to seek is that it should belong to $L^{q}$ for some exponent $q$ strictly less than one. The case $q<1$ is more subtle than $q \geq 1$, and has Diophantine aspects. Such subtleties arise neither for linear operators, nor for the bilinear case, $m=2$, which amounts to ordinary convolution after a change of variables; $L^{1} \otimes L^{1}$ is mapped to $L^{1}$. We will comment briefly on the case $m>3$, which presents further complications and is not yet resolved.

Standard arguments fail to apply when $q<1$. For instance, since $L^{q}$ is not locally convex, one cannot dualize to convert the problem to estimation of a multilinear scalar-valued form $I\left(f_{0}, f_{1}, f_{2}, f_{3}\right)=\int T\left(f_{1}, f_{2}, f_{3}\right)(x) f_{0}(x) d x$;

Received August 26, 2000, revised January 24, 2001.

The author was supported in part by NSF grant DMS-9970660 and carried out part of this research while on appointment as a Miller Research Professor in the Miller Institute for Basic Research in Science. 
rearrangement inequalities such as Theorem 3.8 of [11] are thus of no use here. Indeed, we will see that in opposition to the behavior of multilinear forms $I$, symmetric nondecreasing functions are far from being extremal for multilinear operators (1).

This problem was posed by Kenig and Stein [8], who analyzed more singular operators of fractional integral type, but whose analysis relied substantially on inequalities for less singular operators such as (1). Their results included global estimates in $L^{q}$ for some $q<1$, but they worked in a range of exponents $p$ for which $L^{p} \otimes \cdots \otimes L^{p}$ is mapped to $L_{\mathrm{loc}}^{1}$, as is never the case for (1) when $m=3, p<3 / 2$. Overlapping results were obtained by Grafakos and Kalton [3].

A second motivation was the work of Lacey and Thiele $[9,10]$ on a still more singular operator, the bilinear Hilbert transform; their investigations have to date left open the question of boundedness when the target exponent $q$ is $\leq 2 / 3$. Moreover, very little is known concerning trilinear analogues such as $\int_{\mathbb{R}} f(x-t) g(x+t) h(x-\theta t) t^{-1} d t$. Unfortunately, our analysis seems not to be directly relevant to either of those issues. A final motivation is that our analysis makes strong contact with both recent $[1,7]$ and ancient $[6]$ investigations of the Kakeya problem.

Definition. Suppose that $\left\{S_{j}\right\}$ is nondegenerate. The collection of linear mappings $\left\{S_{j}: 0 \leq j \leq 3\right\}$ is said to be rationally commensurate if there exist linear automorphisms $h_{j}$ of $\mathbb{R}^{d}$ such that the vector subspace of endomorphisms of $\mathbb{R}^{2}$ generated by $\mathbb{Q}$-linear combinations of $\left\{h_{j} \circ S_{j}: 0 \leq j \leq 3\right\}$ has dimension two over $\mathbb{Q}$.

Theorem 1. Suppose that $\left\{S_{j}\right\}$ is nondegenerate. If $\left\{S_{j}\right\}$ is rationally commensurate, then there exists $p<3 / 2$ such that the trilinear operator

$$
\int_{\mathbb{R}^{d}} \prod_{j=1}^{3} f_{j}\left(S_{j}(x, t)\right) d t
$$

maps $L^{p} \otimes L^{p} \otimes L^{p}$ boundedly to $L^{q}$, where $1+q^{-1}=3 p^{-1}$.

If on the other hand $\left\{S_{j}\right\}$ is not rationally commensurate, then for any $p<$ $3 / 2$, there exist nonnegative functions $f_{j} \in L^{p}$ and a set $E \subset \mathbb{R}^{d}$ of positive Lebesgue measure such that $T\left(f_{1}, f_{2}, f_{3}\right)(x)=+\infty$ for all $x \in E$.

Given any $p<3 / 2$, there exist rationally commensurate $\left\{S_{j}\right\}$, nonnegative functions $f_{j} \in L^{p}$, and a set $E$ as above, such that for all $x \in E$, $T\left(f_{1}, f_{2}, f_{3}\right)(x)=+\infty$.

For any $\left\{S_{j}\right\}$ there exist $p>1$, functions $f_{j} \in L^{p}$, and a set $E$ as above, such that $T\left(f_{1}, f_{2}, f_{3}\right)(x)=+\infty$ for all $x \in E$.

The integral defining the operator is taken over all of $\mathbb{R}^{d}$ in the first conclusion, but over a bounded region in the other statements.

At least two interesting questions remain unresolved. Firstly, what is the optimal range of exponents $p$ for each rationally commensurate $\left\{S_{j}\right\}$ ? Secondly, what is the situation for multilinear operators of higher order? Although the 
trilinear analysis settles many higher order cases, the generic case remains open; see $\S 7$ for some discussion. To obtain sharper bounds for certain higher-order operators might lead to improved estimates on the Kakeya problem [7].

The first and third parts of Theorem 1 may be equivalently reformulated in a discrete setting; this is carried out in $\S 5$. We will discuss the one-dimensional case first, leaving the simple modification for $d>1$ to $\S 6$.

\section{The irrational case}

We begin with the one-dimensional case

$$
T\left(f_{1}, f_{2}, f_{3}\right)(x)=\int_{-1}^{1} \prod_{j=1}^{3} f_{j}\left(\nu_{j} \cdot(x, t)\right) d t
$$

where the $\nu_{j} \in \mathbb{R}^{2}$ are nonzero vectors, $\nu_{0}=(1,0),(x, t) \in \mathbb{R}^{2}$, and no two elements of the set $\left\{\nu_{j}: 0 \leq j \leq 3\right\}$ are linearly dependent.

Simple symmetries can be used to reduce the multilinear operator $T$ to a canonical form. One type of symmetry is to replace $f_{j}$ by $\tilde{f}_{j}(s)=f_{j}\left(\lambda_{j} s\right)$ for each $j$, with $\lambda_{j} \neq 0$. $t$ may be replaced by any linear combination $\tilde{t}$ of $(x, t)$, so long as the transformation $(x, t) \mapsto(x, \tilde{t})$ is nonsingular. And $x$ may be replaced by $\lambda_{0} x$. By choosing $\lambda_{1}, \lambda_{2}$ so that $\left(\lambda_{1} \nu_{1}+\lambda_{2} \nu_{2}\right) \cdot(x, t)=2 x$, then setting $\tilde{t}=\left(\lambda_{1} \nu_{1}-\lambda_{2} \nu_{2}\right) \cdot(x, t)$, and finally introducing $\lambda_{3}$ as needed, we may reduce to $T(F)=\int f_{1}(x+t) f_{2}(x-t) f_{3}(x-\theta t) d t$ for some parameter $\theta \in \mathbb{R} \backslash\{0,-1,+1\}$, or to $\int f_{1}(x+t) f_{2}(x-t) f_{3}(t) d t$.

Such transformations may alter the endpoints \pm 1 of the interval of integration, but it is easily verified that no such alteration has any effect on the boundedness of the operator. Moreover, the notion of rational commensurability is invariant under such transformations. The operator $\int f_{1}(x+t) f_{2}(x-t) f_{3}(t) d t$ corresponds to a rationally commensurate set of vectors $\nu_{j}$, while $\int f_{1}(x+t) f_{2}(x-t) f_{3}(x-$ $\theta t) d t$ corresponds to a rationally commensurate set if and only if $\theta \in \mathbb{Q}$.

Let $\theta$ be irrational, and consider $T_{\theta}(f, g, h)(x)=\int_{-1}^{1} f(x+t) g(x-t) h(x-$ $\theta t) d t$. Then (see Theorem 185 of [4]) the continued fraction expansion of $(1+$ $\theta) /(1-\theta)$ generates sequences $\left\{p_{n}\right\},\left\{q_{n}\right\}$ of integers tending to $\infty$, such that $p_{n}, q_{n}$ are relatively prime for each $n$, and so that

$$
\left|\frac{1+\theta}{1-\theta}-\frac{p_{n}}{q_{n}}\right|<\frac{1}{q_{n}^{2}} .
$$

The exponent 2 on the right-hand side will be essential to the construction. Henceforth we drop the subscript $n$, writing simply $p, q$; all assertions are to be uniform in $n$.

Set $N=q$ and $\delta=C_{1} N^{-2}$, where $C_{1}$ is a sufficiently small constant. Thus $N \rightarrow \infty$ as $n \rightarrow \infty$. 
Let $f, g, h$ respectively be the characteristic functions of the following sets $F, G, H$ :

$$
\begin{aligned}
F & =\bigcup_{j=1}^{p}\left\{x:\left|x-j p^{-1}\right|<\delta\right\}, \\
G & =\bigcup_{k=1}^{q}\left\{\left|x-k q^{-1}\right|<\delta\right\}, \\
H & =\bigcup_{l=1}^{p+q}\left\{|x-l y|<C_{2} \delta\right\},
\end{aligned}
$$

where $y=(1-\theta) / 2 q$, and $C_{2}$ is a large constant to be chosen later. Thus $f=\sum_{j=1}^{p} f_{j}$, where each $f_{j}$ is the characteristic function of the $j$-th component interval of $F$; likewise $g=\sum g_{k}$ and $h=\sum h_{l}$.

Since $N \rightarrow \infty$ and $N^{2} \delta \sim 1, N \delta \rightarrow 0$ as $N \rightarrow \infty$. Each of our three functions $f, g, h$ has $L^{p}$ norm $\sim(N \delta)^{1 / p} \sim N^{-1 / p}$.

Observe that $T_{\theta}\left(f_{j}, g_{k}, 1\right)$ has size $\sim \delta$ on the interval of length $\delta$ centered at $\left(j p^{-1}+k q^{-1}\right) / 2$, and is supported on the concentric interval with length $4 \delta$.

We claim two things. First, the $p \cdot q$ functions $T_{\theta}\left(f_{j}, g_{k}, 1\right)$ have pairwise disjoint supports ${ }^{1}$. This implies that $T_{\theta}(f, g, 1) \sim \delta$ at every point of a set $\mathcal{E}_{N}$ of measure $\gtrsim N^{2} \delta \sim 1$. Moreover, the sets $\mathcal{E}_{N}$ are subsets of a fixed compact subset of $\mathbb{R}$, independent of $N$.

It is convenient to modify the definition of $T_{\theta}$ by replacing the limits of integration \pm 1 by $\pm M$ for a large but fixed constant $M$; by scaling, it suffices to prove the stated result for this operator. The second claim is then that $T_{\theta}(f, g, h) \equiv T_{\theta}(f, g, 1)$, provided $M$ is sufficiently large. Thus

$$
T_{\theta}(f, g, h)(x) /\|f\|_{p}\|g\|_{p}\|h\|_{p} \gtrsim \delta(N \delta)^{-3 / p} \sim N^{\frac{3}{p}-2}
$$

for all $x \in \mathcal{E}_{N}$, for all $N$. If $p<3 / 2$ then this exponent $\frac{3}{p}-2$ is positive.

To verify the first claim we need only note that the points $j p^{-1}+k q^{-1}$ are separated by more than $8 \delta$ for distinct pairs of indices $j, k$. This holds because $p, q$ are relatively prime, whence $\left|a p^{-1}+b q^{-1}\right| \geq(p q)^{-1} \sim N^{-2}$ whenever $(a, b) \neq$ $(0,0)$.

To verify the second claim it suffices to show that there exists $C_{2}$ such that whenever $x+t=j p^{-1}$ and $x-t=k q^{-1}$, there exists an integer $l$ such that $|(x-\theta t)-l y| \leq C_{2} \delta$. Now

$$
\begin{aligned}
(x-\theta t) & =\frac{1}{2}\left[(1+\theta) j p^{-1}+(1-\theta) k q^{-1}\right] \\
& =\frac{1}{2}(1-\theta)(j+k) q^{-1}+j p^{-1} \cdot O\left(N^{-2}\right)=(j+k) y+O\left(N^{-2}\right),
\end{aligned}
$$

so it suffices to set $l=j+k$.

\footnotetext{
${ }^{1}$ Since there are $\sim N^{2}$ such functions, each of whose supports is an interval of length $\sim \delta$, and since these are all supported in a fixed set independent of $n$, a necessary condition for this disjointness is that $N^{2} \delta \lesssim 1$; that explains the choice $\delta \sim N^{-2}$.
} 
To complete the proof is now a straightforward matter. Abusing notation, we let $\left\{N_{\nu}\right\}$ be a subsequence of the sequence $\left\{N_{n}\right\}$ constructed above, satisfying $N_{\nu} \geq 2^{\nu}$. Denote by $f^{\nu}, g^{\nu}, h^{\nu}$ the associated functions constructed above, and write $\mathcal{E}_{\nu}$ for the associated sets on which $T_{\theta}\left(f^{\nu}, g^{\nu}, h^{\nu}\right)$ is large. Fix $p<$ $3 / 2$, and choose a sequence $\left\{c_{\nu}\right\}$ of positive constants such that $\sum_{\nu} c_{\nu}<\infty$, but $c_{\nu} 2^{\nu\left(\frac{1}{p}-\frac{2}{3}\right)} \rightarrow \infty$. Define $f=\sum_{\nu} c_{\nu} N_{\nu}^{1 / p} f^{\nu}, g=\sum_{\nu} c_{\nu} N_{\nu}^{1 / p} g^{\nu}$, and $h=$ $\sum_{\nu} c_{\nu} N_{\nu}^{1 / p} h^{\nu}$. Thus $f, g, h \in L^{p}$. Moreover, $T_{\theta}(f, g, h) \geq \sum_{\nu} c_{\nu}^{3} T_{\theta}\left(f^{\nu}, g^{\nu}, h^{\nu}\right)$, so that $T_{\theta}(f, g, h)(x) \geq c_{\nu}^{3} 2^{\nu\left(\frac{3}{p}-2\right)}$ for all $x \in \mathcal{E}_{\nu}$, for every $\nu$. Since all the sets $\mathcal{E}_{\nu}$ are contained in a fixed compact set, and their measures are bounded below by a uniform positive constant, the set of all $x$ belonging to infinitely many sets $\mathcal{E}_{\nu}$ has positive measure. Since $c_{\nu}^{3} 2^{\nu\left(\frac{3}{p}-2\right)} \rightarrow \infty$, the proof is complete.

\section{The rationally commensurate case}

The proof for the rationally commensurate case is an adaptation from the discrete to the continuum setting of an argument of Katz and Tao [7], who sharpened an estimate of Bourgain [1]. Their result directly rules out the existence of any examples resembling those constructed in the preceding section, but does not seem to directly imply any positive $L^{p}$ inequalities. Instead, we will deduce from the method of proof in [7] continuum analogues of variants of its conclusion ${ }^{2}$.

We continue to assume that $d=1$. It suffices to show that there exist $\delta \in(0,1)$ and $0<r_{1}, r_{2}, s$, depending on $\left\{\nu_{j}\right\}$ and satisfying $r_{1}+r_{2}+s=2-\delta$, such that for any measurable sets $A, B, C \subset \mathbb{R}$ and any $\lambda>0$, the set $E=\{x$ : $\left.T\left(\chi_{A}, \chi_{B}, \chi_{C}\right)(x)>\lambda\right\}$ satisfies

$$
\lambda^{1-\delta}|E| \lesssim|A|^{r_{1}}|B|^{r_{2}}|C|^{s} .
$$

Indeed, $T$ maps $L^{2} \otimes L^{2} \otimes L^{1}$ boundedly to $L^{1}$, and so there is always the relatively trivial bound

$$
\lambda|E| \lesssim \min \left(|A|^{1 / 2}|B|^{1 / 2}|C|^{1},|A|^{1 / 2}|C|^{1 / 2}|B|^{1},|B|^{1 / 2}|C|^{1 / 2}|A|^{1}\right) .
$$

Raising (3) to a power $\theta \in[0,1]$ and multiplying by any powers, summing to $1-\theta$, of the three permutations of (4), produces another inequality of the form (3). Choosing the powers appropriately yields an inequality (3) with $r_{1}=r_{2}=s$, albeit with a smaller $\delta$. Writing it as a restricted weak type estimate $\lambda^{q_{0}}|E| \leq$ $(|A| \cdot|B| \cdot|C|)^{\frac{q_{0}}{p_{0}}}$, we find that $q_{0}<1$ and $\frac{3 q_{0}}{p_{0}}=1+q_{0}$, whence $p_{0}<3 / 2$. From multilinear interpolation $[5,8]$ we then conclude easily that for any $p>p_{0}, T$ maps the threefold tensor product of $L^{p}$ boundedly to $L^{q}$ where $\frac{3}{p}=1+\frac{1}{q}$.

In an attempt to make the exposition more easily digestible, we will discuss the special case $T(f, g, h)(x)=\int_{-\infty}^{\infty} f(x+t) g(x-t) h(t) d t$ first. Here the argument

\footnotetext{
${ }^{2}$ The conclusion of Katz, Tao, and Bourgain concerns the cardinality of a certain image set; the variant we require also takes into account cardinalities of sets of preimages. Compare Theorem 2 with its corollary, below.
} 
is based rather directly on [7]. By replacing $B$ by $-B, C$ by $\frac{1}{2} C=\{s / 2: s \in C\}$, and $E$ by $\frac{1}{2} E$, we may arrange that for each $x \in E$,

$$
\mid\{t \in C: t+x \in 2 A \text { and } t-x \in 2 B\} \mid \geq \lambda .
$$

Let

$$
\tilde{G}=\{(a, b) \in A \times B: a+b \in C \text { and } a-b \in E\} .
$$

Select a measurable subset $G \subset \tilde{G}$ such that for each $x \in E, \mid\{(a, b) \in G$ : $a-b=x\} \mid=\lambda$, and denote the latter set by $G_{x}$. Here the measure in question is one-dimensional Lebesgue measure on a line, determined by $x$, in $\mathbb{R}^{2}$. From this and the definition of $\tilde{G}$, it follows that

$$
|G|=\lambda|E| \text {. }
$$

Define

$$
V=\left\{\left(a, b, b^{\prime}\right) \in \mathbb{R}^{3}:(a, b) \in G \text { and }\left(a, b^{\prime}\right) \in G\right\}
$$

Then

$$
|V| \geq|G|^{2} /|A| \text {. }
$$

Indeed, consider the map $\pi: G \mapsto A$ defined by $\pi(a, b)=a$. Set $\rho(a)=\left|\pi^{-1}\{a\}\right|$, for $a \in A$, where $|\cdot|$ denotes one-dimensional measure on the fibers. Then

$$
|V|=\int_{A} \rho(a)^{2} d a \geq|A|^{-1}\left(\int_{A} \rho\right)^{2}=|A|^{-1}|G|^{2} .
$$

Define $S \subset V^{4}$ to be the set of all points $\left(v_{0}, v_{1}, v_{2}, v_{3}\right)$ satisfying all of the following relations ${ }^{3}$

$$
\begin{aligned}
a_{0}+b_{0} & =a_{1}+b_{1}, & a_{0}+b_{0}^{\prime} & =a_{1}+b_{1}^{\prime}, \\
b_{1} & =b_{2}, & b_{1}^{\prime} & =b_{2}^{\prime}, \\
a_{2}+b_{2} & =a_{3}+b_{3}, & b_{2}^{\prime} & =b_{3}^{\prime} .
\end{aligned}
$$

$S$ is contained in a linear subspace of $\mathbb{R}^{12}$ of dimension 6 . As coordinates for $S$, we use $V \times A \times B^{2} \ni\left(v_{0}, a_{2}, b_{3}, b_{3}^{\prime}\right)=\left(a_{0}, b_{0}, b_{0}^{\prime}, a_{2}, b_{3}, b_{3}^{\prime}\right)$. It follows from the discussion in [7] that all other coordinates $a_{j}, b_{j}, b_{j}^{\prime}$ of any point of $S$ may be determined from these six coordinates by various linear relations. Thus $|S| \lesssim$ $|A| \cdot|B|^{2} \cdot|V|$.

By reasoning similar to that in Lemma 2.1 of [7] and the above discussion of (9), we find that

$$
|S| \gtrsim|V|^{4} /|B|^{3}|C|^{3}
$$

The details are left to the reader.

\footnotetext{
${ }^{3}$ There is a typographical error in the last equation of formula (15) in [7], which should $\operatorname{read} b_{1}^{\prime}=b_{2}^{\prime}$.
} 
The key point of the proof is that a special algebraic relation holds, allowing a sharpening of the upper bound on $|S|$, as follows. It is shown in [7] that if $\left(v_{0}, v_{1}, v_{2}, v_{3}\right)=s \in S$, then

$$
a_{3}-b_{3}^{\prime}=a_{2}+b_{0}-b_{0}^{\prime}-b_{3} .
$$

Thus once the coordinates $a_{2}, b_{0}, b_{0}^{\prime}, b_{3}$ of a point $s$ of $S$ are known, $a_{3}-b_{3}^{\prime}$ can be computed. But since $\varphi(s)=\left(a_{3}, b_{3}^{\prime}\right)$ belongs to $G$ by construction, $\varphi(s) \in G_{x}$, where $x=a_{2}+b_{0}-b_{0}^{\prime}-b_{3}$. Projecting $\varphi(v)$ onto its second coordinate, we conclude that for any $\left(a_{2}, b_{0}, b_{0}^{\prime}, b_{3}\right)$, for any $s \in S$ sharing these four coordinates, the coordinate $b_{3}^{\prime}$ of $s$ must belong to the projection of $G_{x}$ onto the $b_{3}^{\prime}$ axis, and the measure of this projection is $\leq \sqrt{2} \cdot \lambda$. Thus we arrive at the crucial estimate

$$
|S| \lesssim \lambda|A| \cdot|B| \cdot|V| \text {. }
$$

Combining (11) with (13), we deduce that

$$
\lambda|A| \cdot|B| \cdot|V| \gtrsim|V|^{4}|B|^{-3}|C|^{-3},
$$

so

$$
|V| \lesssim \lambda^{1 / 3}|A|^{1 / 3}|B|^{4 / 3}|C|
$$

Since $|V| \gtrsim|G|^{2} /|A|$, this yields

$$
|G| \lesssim \lambda^{1 / 6}|A|^{2 / 3}|B|^{2 / 3}|C|^{1 / 2}
$$

Since $|G|=\lambda|E|$, this can be rewritten as

$$
\lambda^{5 / 6}|E| \lesssim|A|^{2 / 3}|B|^{2 / 3}|C|^{1 / 2}
$$

If the roles of $A, B, C$ could be symmetrized, this would then yield $\lambda^{5 / 6}|E| \lesssim$ $(|A| \cdot|B| \cdot|C|)^{11 / 18}$, which would yield exponents $p=15 / 11$ and $q=5 / 6$ in our final conclusion. But such a symmetrization does not seem to follow directly from the above setup, and since there is little reason to believe that $p=15 / 11$ is optimal, we have not labored to obtain it. Instead, the discussion of (3) yields a conclusion of the desired form, for all $p>7 / 5$. This concludes the discussion of the simplest case in which estimates do hold with $p<3 / 2$.

In the general rationally commensurate case, one has nonzero integers $m, k$ such that $c=a+b \in C$ and $e=m a+k b \in E$ whenever $(a, b) \in \tilde{G}$. Moreover, $(m, k) \in \mathbb{Z}^{2}$ is not a scalar multiple of $(1,1)$. By replacing $e$ by a constant multiple, we may assume $m, k$ to be relatively prime. We may also suppose them to have opposite signs. If for instance both are positive and $m>k$, then we interchange the roles of the sets $B, C$, replace $a$ by $\tilde{a}=-a$, and express $b, e$ in terms of $(\tilde{a}, c)$ by $b=c-a=\tilde{a}+c$ and $e=m a+k b=(k-m) \tilde{a}+k c$, producing coefficients with opposite signs. Other cases are similar. Thus we need only consider the situation where $m, k$ are strictly positive and relatively prime.

Letting $L, L^{\prime}$ be nonnegative integers to be chosen below, let $V$ be the set of all $\left(L+L^{\prime}+2\right)$-tuples $^{4}\left(a^{0}, \ldots, a^{L}, b^{0}, \ldots, b^{L^{\prime}}\right)$ such that $\left(a^{i}, b^{j}\right) \in G$ for all such $i, j \geq 0$. $V$ is a subset of a Euclidean space of dimension $L+L^{\prime}+2$. To estimate

\footnotetext{
${ }^{4}$ These superscripts are not exponents.
} 
the Lebesgue measure of $V$, define $V_{0}$ to be the set of all $\left(a, b^{0}, \ldots, b^{L^{\prime}}\right)$ such that $\left(a, b^{j}\right) \in G$ for all $0 \leq j \leq L^{\prime}$. Consider the Cartesian product $V_{0}^{L+1}$, give it coordinates $\left(a_{0}, b_{0}^{0}, \ldots, b_{0}^{L^{\prime}}\right), \ldots,\left(a_{L}, b_{L}^{0}, \ldots, b_{L}^{L^{\prime}}\right)$, and regard $V$ as the set of all points in $V_{0}^{L+1}$ satisfying the $L\left(L^{\prime}+1\right)$ equations $b_{n}^{j}=b_{n-1}^{j}$ for all $j$ and all $1 \leq n \leq L$. As in (9), $\left|V_{0}\right| \geq|G|^{L^{\prime}+1}|A|^{-L^{\prime}}$. Thus

$$
|V| \gtrsim\left|V_{0}\right|^{L+1}|B|^{-L\left(L^{\prime}+1\right)} \gtrsim|G|^{(L+1)\left(L^{\prime}+1\right)}|A|^{-L^{\prime}(L+1)}|B|^{-L\left(L^{\prime}+1\right)} .
$$

Setting $M=L+L^{\prime}$, we define $S \subset V^{M+3}$ to be the set of all $\left(v_{M+2}, \ldots, v_{0}\right)$ satisfying certain linear relations. For each $1 \leq n \leq M+2$ we will impose $L+L^{\prime}+1=\operatorname{dim}(V)-1$ linear relations, linking $v_{n}$ to $v_{n-1}$. For each $n=$ $M+2, M+1, \ldots, 3$, one of only two types of collections of relations will be used.

$$
\text { Type I: } \quad\left\{\begin{aligned}
a_{n}^{i}+b_{n}^{s} & =a_{n-1}^{i}+b_{n-1}^{s} & & \text { for all } i, \\
b_{n}^{j} & =b_{n-1}^{j} & & \text { for all } j \neq s,
\end{aligned}\right.
$$

where $s \leq L^{\prime}$ is any nonzero index, depending on $n$. The second type is the same, but with the roles of the $a$ and $b$ variables interchanged.

$$
\text { Type II: } \quad\left\{\begin{aligned}
a_{n}^{t}+b_{n}^{j} & =a_{n-1}^{t}+b_{n-1}^{j} & & \text { for all } j, \\
a_{n}^{i} & =a_{n-1}^{i} & & \text { for all } i \neq t,
\end{aligned}\right.
$$

where $t \leq L$ is any nonzero index, depending on $n$. Each superscript $s, t$ will be used in this way exactly once; in aggregate over all $2 \leq n \leq M+2$, the Type I will relations will be used $L^{\prime}$ times, and the Type II relations, $L$ times. A useful consequence of these relations is that for all $n$,

$$
a_{n}^{i}-a_{n}^{0}=a_{n-1}^{i}-a_{n-1}^{0} \text { and } b_{n}^{j}-b_{n}^{0}=b_{n-1}^{j}-b_{n-1}^{0}
$$

for all $i, j$, except for $i=t$ in the Type II case, and for $j=s$ in the Type I case. The cases $n=2,1$ will be different and will be discussed later.

In order to explain which of the two types of sets of relations is employed at each step, we begin with the calculation dictating their selection. Suppose without loss of generality that $m<k$. Our ultimate goal is an analogue of (12), expressing $m a_{n}^{0}-k b_{n}^{0}$ in terms of (other) coordinates, still to be selected, for $S$. The first step in deriving such a representation is to write

$$
\begin{aligned}
{\left[m a_{M+2}^{0}-k b_{M+2}^{0}\right] } & =m a_{M+2}^{0}+m b_{M+2}^{1}-k b_{M+2}^{0}-m b_{M+2}^{1} \\
& =m\left(a_{M+1}^{0}+b_{M+1}^{1}\right)-k b_{M+1}^{0}-m b_{M+2}^{1} \\
& =\left[m a_{M+1}^{0}-(k-m) b_{M+1}^{0}\right]+m\left(b_{M+1}^{1}-b_{M+1}^{0}\right)-m b_{M+2}^{1} .
\end{aligned}
$$

This effectively reduces the pair $(m, k)$ to $(m, k-m)$, as will be shown below. To pass from the first line to the second we have used (a subset of) the Type I relations, with $s=1$.

Since $m, k$ are relatively prime, so are $m, k-m$. Which of the two types is used at the next step depends on which of $m, k-m$ is larger. If $k-m<m$, we let $\tilde{k}=k-m$, reverse the roles of the $a, b$ variables, and employ the Type II 
relations, which permit us to write the main term remaining after the preceding step as

$$
\begin{aligned}
{\left[m a_{M+1}^{0}-\tilde{k} b_{M+1}^{0}\right] } & =-\tilde{k}\left(a_{M+1}^{1}+b_{M+1}^{0}\right)+m a_{M+1}^{0}+\tilde{k} a_{M+1}^{1} \\
& =-\tilde{k}\left(a_{M}^{1}+b_{M}^{0}\right)+m a_{M}^{0}+\tilde{k} a_{M+1}^{1} \\
& =\left[(m-\tilde{k}) a_{M}^{0}-\tilde{k} b_{M}^{0}\right]+\tilde{k}\left(a_{M}^{0}-a_{M}^{1}\right)+\tilde{k} a_{M+1}^{1} .
\end{aligned}
$$

The variable $a_{M+1}^{1}$ will serve as one of the coordinates for the ambient vector space in which $S$ sits, as will $b_{M+2}^{1}$. From the first step, there remains $m\left(b_{M+1}^{1}-\right.$ $\left.b_{M+1}^{0}\right)$, which iequals $m\left(b_{M}^{1}-b_{M}^{0}\right)$ by (17). If on the other hand $k-m>m$, then we use the Type I relations again.

Thus in the subcase where $k-m<m<k$, after two steps we have

$$
m a_{M+2}^{0}-k b_{M+2}^{0}=\tilde{m} a_{M}^{0}-\tilde{k} b_{M}^{0}
$$

modulo a linear combination of two coordinates $b_{M+2}^{1}, a_{M+1}^{1}$, and modulo a linear combination of two differences, $b_{M}^{1}-b_{M}^{0}$ and $a_{M}^{1}-a_{M}^{0}$. Here $\tilde{m}, \tilde{k}$ are nonnegative, are $\leq m, k$, respectively, and at least one of them is positive. If both are positive, then they are relatively prime, and at least one is strictly less than $m, k$, respectively.

This process is repeated until $m a_{M+2}^{0}-k b_{M+2}^{0}$ equals a multiple of either $a_{2}^{0}$ or $b_{2}^{0}$, modulo a linear combination of differences $b_{2}^{j}-b_{2}^{0}$ and $a_{2}^{i}-a_{2}^{0}$, and modulo a linear combination of all the coordinates $a_{n}^{i}, b_{n}^{j}$, with $1 \leq i \leq L, 1 \leq j \leq L^{\prime}$. The only change is that the role of the superscript 1 is taken by larger numbers $s, t$. This process generates exactly one coordinate variable, either $a_{n}^{i}$ or $b_{n}^{j}$, for each $3 \leq n \leq M+2=L+L^{\prime}+2$. At each step $n$, any difference $b_{n}^{j}-b_{n}^{0}$ remaining from the preceding step $n+1$ can be expressed as $b_{n-1}^{j}-b_{n-1}^{0}$, by (17); such indices $j$ arising after step $n+1$ will never equal the index $s$ used in the Type I relations at step $n$, so that (17) does apply. Likewise for differences $a_{n}^{i}-a_{n}^{0}$.

The numbers $L, L^{\prime}$ are determined by the condition that upon starting with $n=M+2=L+L^{\prime}+2$, this procedure reduces $m a_{M+2}^{0}-k b_{M+2}^{0}$ to a multiple of $a_{2}^{0}$ or $b_{2}^{0}$ at $n=2$.

Suppose that we are in the subcase where $m a_{M+2}^{0}-k b_{M+2}^{0}$ equals a multiple of $b_{2}^{0}$ modulo the other terms just described; the case of $a_{2}^{0}$ is completely parallel. Then we link $v_{2}$ to $v_{1}$ by imposing the relations $b_{2}^{j}=b_{1}^{j}$ for all $j \geq 0$, and $a_{2}^{i}=a_{1}^{i}$ for all $i \geq 1$. Finally, we link $v_{1}$ to $v_{0}$ by $a_{1}^{0}+b_{1}^{j}=a_{0}^{0}+b_{0}^{j}$ for all $j \geq 0$, and $a_{1}^{i}=a_{0}^{i}$ for all $i \geq 1$.

In this subcase, as coordinates for the ambient vector space we take for each step $3 \leq n \leq M+2$ either some $a_{n}^{t}$ or $b_{n}^{s}$, as described above, giving $M=L+L^{\prime}$ coordinates. To these we adjoin $v_{0}, a_{2}^{0}$ and $b_{M+2}^{0}$. The various other cases and subcases are similar, and the details are left to the reader. The total number of coordinates is $L+L^{\prime}+\left(L+L^{\prime}+2\right)+1+1=2\left(L+L^{\prime}+2\right)$. Since $V^{L+L^{\prime}+3}$ has dimension $\left(L+L^{\prime}+3\right)\left(L+L^{\prime}+2\right)$ and we have imposed $\left(L+L^{\prime}+2\right)\left(L+L^{\prime}+1\right)$ relations, there should indeed be $2\left(L+L^{\prime}+2\right)$ coordinates. We thus have 
a key identity expressing $m a_{M+2}^{0}-k b_{M+2}^{0}$ as a linear combination of all the coordinates, excluding $b_{M+2}^{0}$ itself, modulo the relations.

Assuming that these coordinate variables do form a valid coordinate system, we find by calculations analogous to those in the special case $(m, k)=(1,1)$ that $|S| \gtrsim|V|^{L+L^{\prime}+3}|A|^{-r_{1}}|B|^{-r_{2}}|C|^{-r_{3}}$ for certain positive integer exponents $r_{l}$ whose sum $r_{1}+r_{2}+r_{3}$ equals the total number of relations, $\left(L+L^{\prime}+2\right)\left(L+L^{\prime}+1\right)$. There is also a lower bound, $|S| \lesssim \lambda|A|^{s}|B|^{s^{\prime}}|V|$ where $s+s^{\prime}=L+L^{\prime}+1$. Together with (16), these lead to an inequality of the form (3), as desired.

It remains to verify that any of the variables $a_{n}^{i}, b_{n}^{j}$ can be expressed as an appropriate linear combination of the $2\left(L+L^{\prime}+2\right)$ coordinates described, modulo the relations imposed. $b_{M+2}^{0}$ is a coordinate, and $a_{M+2}^{0}$ can be calculated in terms of coordinates, as a consequence of the key identity. We claim, by descending induction on $n=M+1, M, \ldots, 2$, that $a_{n}^{0}, b_{n}^{0}$ may each be expressed as a linear combination of coordinates and of differences $\left(b_{n}^{j}-b_{n}^{0}\right),\left(a_{n}^{i}-a_{n}^{0}\right)$. Indeed, there is for each $n$ a formula of the general type $\tilde{m} a_{n+1}^{0}-\tilde{k} b_{n+1}^{0}=m^{\prime} a_{n}^{0}-k^{\prime} b_{n}^{0}$ modulo coordinates and differences $\left(b_{n}^{j}-b_{n}^{0}\right),\left(a_{n}^{i}-b_{n}^{i}\right)$, along with one of the two relations $b_{n+1}^{0}=b_{n}^{0}$ or $a_{n+1}^{0}=a_{n}^{0}$. Moreover, (17) always gives $a_{n}^{i}-a_{n}^{0}=a_{n-1}^{i}-a_{n-1}^{0}$ and $b_{n}^{j}-b_{n}^{0}=b_{n-1}^{j}-b_{n-1}^{0}$ for all $i, j$ arising at step $n$.

Now consider $n=2 . a_{2}^{0}$ is, in this subcase, a coordinate. We have derived an equation expressing $b_{2}^{0}$ in terms of coordinates and of differences $b_{2}^{j}-b_{2}^{0}, a_{2}^{i}-a_{2}^{0}$, with $i, j \geq 1$. The assumed relations linking $v_{2}$ to $v_{1}$ and thence to $v_{0}$ imply that $b_{2}^{j}-b_{2}^{0}=b_{0}^{j}-b_{0}^{0}$ and $a_{2}^{i}-a_{2}^{0}=a_{0}^{i}-a_{0}^{0}$ for $i, j \geq 1$, so all the differences $b_{2}^{j}-b_{2}^{0}, a_{2}^{i}-a_{2}^{0}$ can be expressed ultimately in terms of coordinates. Thus for all $n \geq 2$, each $a_{n}^{0}, b_{n}^{0}$ may be expressed as a linear combination of coordinates. $b_{1}^{0}$ equals $b_{2}^{0}$, which has already been captured, and the relation $a_{1}^{0}+b_{1}^{0}=a_{0}^{0}+b_{0}^{0}$ then determines $a_{1}^{0}$; of course, $a_{0}^{0}, b_{0}^{0}$ are coordinates. With this information in hand, it is straightforward to recover each $v_{n}$ from $v_{0}$ and from the coordinates $a_{n}^{t}, b_{n}^{s}$ by ascending induction on $n$.

\section{The threshold exponent}

In the rationally commensurate case, there exists $p_{0}=p_{0}\left(\nu_{1}, \nu_{2}, \nu_{3}\right)<3 / 2$ such that $T(F)$ is almost everywhere finite whenever each $f_{j} \in L^{p}$ and $p>p_{0}$. Here we will show that the threshold $p_{0}$ can be arbitrarily close to $3 / 2$, via a construction related to Kahane's construction [6] of a Kakeya set, and to certain examples in [7].

Let $r, s$ be any two relatively prime positive integers, and consider the case where $\nu_{1}+\nu_{2}=(1,0)$ and $r^{-1} \nu_{1}+s^{-1} \nu_{2}=\nu_{3}$; thus $(a, b) \in \tilde{G}$ implies that $a+b \in E$ and $r^{-1} a+s^{-1} b \in C$. Consider the following sets, which depend on $r, s$ and on a large parameter $K$. Let $A=\left\{\sum_{n=1}^{K} a_{n}(r s)^{-n}+z\right\}$ where each $a_{n} \in$ $\{0, r, 2 r, \ldots,(s-1) r\}$ and $|z| \leq 2(r s)^{-K}$ and likewise $B=\left\{\sum_{n=1}^{K} b_{n}(r s)^{-n}+z\right\}$ where each $b_{n} \in\{0, s, 2 s, \ldots,(r-1) s\}$ and $\left.|z| \leq 2(r s)^{-K}\right\}$. Set $C=r^{-1} A+$ $s^{-1} B$. Then $|A| \sim s^{K}(r s)^{-K}=r^{-K}$, and likewise $|B| \sim s^{-K}$. Finally, any element of $C$ may be expressed as $\sum_{n=1}^{K} c_{n}(r s)^{-n}+z$ where $0 \leq c_{n} \leq r+s-$ 
2 is an integer, and $|z| \leq 4(r s)^{-K}$. Thus $|C| \lesssim(r+s)^{K}(r s)^{-K}$. We have $T\left(\chi_{A}, \chi_{B}, \chi_{C}\right)(x) \gtrsim(r s)^{-K}$ whenever $x$ is within distance $(r s)^{-K}$ of any sum $\sum_{n=1}^{K}\left(i_{n} r+j_{n} s\right)(r s)^{-n}$ with $0 \leq i_{n}<s$ and $0 \leq j_{n}<r$. Any two such sums are distinct, so the set of all of them has cardinality $(r s)^{K}$, and any two such sums differ by at least $(r s)^{-K}$. So $T\left(\chi_{A}, \chi_{B}, \chi_{C}\right)(x) \gtrsim(r s)^{-K}$ for all $x$ in a set whose measure is bounded below by a constant, uniformly in $r, s, K$. Thus for any $q>0$, the $L^{q}$ norm of $T\left(\chi_{A}, \chi_{B}, \chi_{C}\right)$ is $\gtrsim(r s)^{-K}$, uniformly in $r, s, K$. A necessary condition for an $L^{p} \otimes L^{p} \otimes L^{p} \mapsto L^{q}$ inequality, with $r, s$ fixed, is therefore that

$$
(r s)^{-K} \lesssim r^{-K / p} s^{-K / p}(r+s)^{K / p}(r s)^{-K / p} .
$$

Letting $K \rightarrow \infty$, we find that a necessary condition is that

$$
1 \leq r^{1-\frac{2}{p}} s^{1-\frac{2}{p}}(r+s)^{\frac{1}{p}}
$$

To deduce that $p$ must be arbitrarily close to $3 / 2$, it suffices to choose relatively prime $r, s$ with $s / r$ arbitrarily close to 1 .

As in $\S 2$, it is easy to deduce from the same examples that the threshold exponent $p_{0}$ for almost everywhere finiteness must satisfy the same restriction.

The final conclusion of Theorem 1 follows from the same construction.

\section{Discrete analogues}

The following discrete analogue is a descendant of results of Bourgain [1], and of Katz and Tao [7], and is related to work Gowers [2]. Denote by $|S|$ the cardinality of any finite set $S$.

Theorem 2. Suppose that $(m, k),\left(m^{\prime}, k^{\prime}\right) \in \mathbb{Z}^{2}$ and that no two of the vectors $(1,0),(0,1),(m, k),\left(m^{\prime}, k^{\prime}\right)$ are linearly dependent. Then there exist $p<3 / 2$ and $K<\infty$, depending only on $m, k, m^{\prime}, k^{\prime}$, such that for any torsion-free Abelian group $\mathfrak{G}$, for any finite subsets $A, B, C \subset \mathfrak{G}$, the multiplicity function

$$
\mu(x)=\mid\left\{(a, b, c) \in A \times B \times C: c=m^{\prime} a+k^{\prime} b \text { and } x=m a+k b\right\} \mid
$$

satisfies for every $\lambda>0$

$$
\sum_{x \in \mathfrak{G}} \mu(x)^{q} \leq K|A|^{q / p}|B|^{q / p}|C|^{q / p}
$$

where $1+q^{-1}=3 p^{-1}$.

The relation $1+q^{-1} \leq 3 p^{-1}$ is necessary in the discrete case, whereas $1+$ $q^{-1} \geq 3 p^{-1}$ is necessary in the continuum setting of Theorem 1 ; for each version, the case of equality $1+q^{-1}=3 p^{-1}$ trivially implies the appropriate cases of inequality.

As noted in [7], whenever (18) holds uniformly for all groups (with $1+q^{-1}=$ $3 p^{-1}$ ), it must actually hold with $K=1$. This is seen by taking $M$-fold products and letting $M \rightarrow \infty$. 
Theorem 2 may be deduced from the argument in $\S 3$ for the continuous case; simple changes of variables reduce matters to the case $\left(m^{\prime}, k^{\prime}\right)=(1,1)$. Alternatively, it is easy to show directly that either of Theorems 1,2 implies the other. For instance, to deduce Theorem 2 from Theorem 1, consider arbitrary finite sets $A, B, C$ in any torsion-free Abelian group. The subgroup generated by them is isomorphic to some $\mathbb{Z}^{d}$; any finite subset of $\mathbb{Z}^{d}$ can be embedded isomorphically into $\mathbb{Z}^{1} \subset \mathbb{R}^{1}$. The resulting image $\tilde{A}$ of $A$ is a collection of points $x_{j} \in \mathbb{Z}^{1}$; define $A^{\prime}=\cup_{j}\left[x_{j}-1, x_{j}+1\right]$. Define $B^{\prime}, C^{\prime}, E^{\prime}$ analogously. The bound for the $L^{q}$ norm of $T\left(\chi_{A^{\prime}}, \chi_{B^{\prime}}, \chi_{C^{\prime}}\right)$ resulting from Theorem 1 can then be converted into a bound for the discrete analogue of $T$, applied to $A, B, C$. The reverse implication uses a limiting argument, in which a discrete set is approximated by its $\delta$-neighborhood, and $\delta \rightarrow 0$. Details are left to the reader.

Corollary. There exists $\delta>0$, depending only on $m, k$, such that for any torsion-free Abelian group $\mathfrak{G}$, any positive integer $N$, and any finite subsets $A, B, C \subset \mathfrak{G}$ such that $|A|,|B|,|C| \leq N$,

$$
\left|\left\{m a+k b:\left(a, b, m^{\prime} a+k^{\prime} b\right) \in A \times B \times C\right\}\right| \leq N^{2-\delta} .
$$

The case where $(m, k)=(1,-1)$ and $\left(m^{\prime}, k^{\prime}\right)=(1,1)$ was treated in [1], and an improved estimate for $\delta$ was obtained in [7]. To deduce the corollary from the preceding theorem, observe that the exponent $\frac{3 q}{p}=\frac{3}{3-p}$ is $<2$.

\section{Higher dimensions}

Consider (1) in arbitrary dimension $d>1$, in the trilinear case $m=3$. By simple changes of variables, we may reduce to the situation in which $E=$ $\{a+\Theta b:(a, b, a+b) \in A \times B \times C\}$, where $\Theta: \mathbb{R}^{d} \mapsto \mathbb{R}^{d}$ is a surjective linear transformation. If $\Theta$ is a rational scalar times the identity mapping, then the analysis of $\S 3$ applies without any changes. Alternatively, this case is a corollary of Theorem 2, by the same limiting argument used to derive Theorem 1 from it in the one-dimensional case.

For purposes of illustration, consider next the special case where $d=2$, and $\Theta=\left(\begin{array}{ll}0 & 1 \\ 1 & 0\end{array}\right)$. Define $A_{N}=B_{N}=C_{N}=\{(j, 0): 1 \leq j \leq 2 N\}$. Then $E \supset\{(i, j): 1 \leq i, j \leq N\}$, so $|E| \geq N^{2}$ while $\left|A_{N}\right|=\left|B_{N}\right|=\left|C_{N}\right|=2 N$. Hence there is no valid bound (19). Nor, in the continuum version, is there boundedness from $L^{p} \otimes L^{p} \otimes L^{p}$ to $L^{q}$ for some $p<3 / 2$, no matter how close $q$ may be to zero.

For the $\mathbb{Z}^{d}$ analogue, we may argue in general as follows. If $\Theta$ is not a scalar multiple of the identity, then there exists $0 \neq v \in \mathbb{R}^{d}$ such that $\Theta(v)$ is not a scalar multiple of $v$. For the discrete analogue, with $A, B, C, E \subset \mathbb{Z}^{d}$, one can then repeat the discussion of the example two paragraphs above, taking $A=B=C=\{j v: 1 \leq j \leq 2 n\}$. For $\mathbb{R}^{d}$, taking $\delta$-neighborhoods of these sets, and considering the limit $\delta \rightarrow 0$, we conclude that there is no $\left(L^{p}\right)^{3} \mapsto L^{q}$ estimate with $1+q^{-1}=3 p^{-1}$. 
For the continuum situation, we note instead that there exists a linear subspace $W \subset \mathbb{R}^{d}$ of dimension $d-1$, such that $W+\Theta(W)=\mathbb{R}^{d}$. Take $B$ to be the unit ball in $W$, and let $A, B, C$ be $\delta$-neighborhoods of $B$. Then $T\left(\chi_{A}, \chi_{B}, \chi_{C}\right)$ is $\geq c \delta^{2}$ in a fixed ball in $\mathbb{R}^{d}$, uniformly in $\delta$. If $T:\left(L^{p}\right)^{3} \mapsto L^{q}$, then $\delta^{2} \leq C \delta^{3 / p}$, whence $p \geq 3 / 2$.

Finally, if $\Theta$ equals an irrational scalar times the identity, then the construction of $\S 2$ applies; now we take for instance $A$ to be a $\delta$-neighborhood of the set of all points $\left(j_{1} p^{-1}, \ldots, j_{d} p^{-1}\right)$ with $1 \leq j_{i} \leq p$ for all $1 \leq i \leq d$.

\section{Remarks}

1. In the rationally commensurate case, we have proved boundedness of $\int_{\mathbb{R}^{d}} \prod_{j} f\left(\nu_{j} \cdot(x, t)\right) d t$, with the integral taken over the whole space $\mathbb{R}^{d}$. Homogeneity then forces the relation $1+q^{-1}=3 p^{-1}$.

2. Homogeneity considerations likewise imply that even when we integrate only over a bounded region of $t$ as in (1), $L^{p} \otimes L^{p} \otimes L^{p}$ is not mapped to $L_{\text {loc }}^{q}$ for any larger value of $q$ (take each $f_{j}$ to be the characteristic function of a small interval $(-\delta, \delta))$. It follows as in [8] and [3] that for all $p>p_{0}, L^{p} \otimes L^{p} \otimes L^{p}$ is mapped to $L^{q}$ whenever it is mapped to $L_{\text {loc }}^{q}$ and $q \geq p / 3$. Thus for all $p>p_{0}$, $T$ maps $L^{p} \otimes L^{p} \otimes L^{p}$ boundedly to $L^{q}$ if and only if $q^{-1} \leq 3 p^{-1} \leq 1+q^{-1}$.

3 . We see no reason to believe that the range of exponents $p$ obtained by our analysis is optimal.

4. The case of higher-order multinearity presents further complications, although certain partial results follow from our analysis of the trilinear case. Given finitely many distinct coefficients $\theta_{j} \neq \pm 1$, consider the multilinear operator

$$
T\left(f, g, h_{1}, \ldots, h_{n}\right)(x)=\int_{-1}^{1} f(x+t) g(x-t) \prod_{j=1}^{n} h_{j}\left(x-\theta_{j} t\right) d t .
$$

It maps the tensor product of $n+2$ copies of $L^{(n+2) / 2}$ to $L^{1}$, and we ask whether it is well-defined on a product of copies of $L^{p}$ for some $p<(n+2) / 2$.

If at least one $\theta_{j}$ is rational, then $T$ is indeed well-defined on $L^{p} \otimes \cdots \otimes L^{p}$ for some $p<(n+2) / 2$, as follows from the corresponding result for trilinear operators. Renaming indices so that $j=1$, we know from the trilinear case that there exist $r<3 / 2$ and $q>0$ such that $L^{r} \otimes L^{r} \otimes L^{r} \otimes L^{\infty} \otimes \cdots \otimes L^{\infty}$ is mapped boundedly to $L^{q}$. An interpolation concludes the proof.

To discuss the irrational case, suppose that $\theta_{1} \notin \mathbb{Q}$. Define the quantities $\gamma_{j}=\left(1+\theta_{j}\right) /\left(1-\theta_{j}\right)$. If all ratios $\gamma_{j} / \gamma_{1}$ are rational, then by the construction in $\S 2$, for any $p<(n+2) / 2$, there exist nonnegative $f, g, h_{1}, \ldots, h_{n} \in L^{p}$ for which $T\left(f, g, h_{1}, \ldots\right)(x)=+\infty$ for all $x$ in a set of positive measure. More generally, certain restrictions on the exponents $(p, q)$ may be deduced in this way, if $\gamma_{1}$ is irrational and there exists an infinite sequence $q_{n} \rightarrow \infty$ such that for each $n, j$ there exists $p_{n, j}$ such that $\left|\gamma_{j}-\frac{p_{n, j}}{q_{n}}\right| \leq C q_{n}^{-2}$, with $C$ independent of $n, j$, and such that the ratios $p_{n, j} / p_{n, k}$ are sufficiently close to being independent 
of $n$ as $n \rightarrow \infty$. The case in which all $\theta_{j}$ are irrational, but no such simultaneous Diophantine approximations exist, remains open.

The examples of $\S 4$ have natural analogues for higher-order operators, with all $\theta_{j} \in \mathbb{Q}$.

5. It is legitimate to ask what might be the optimal estimates of the form $T$ : $L^{p_{1}} \otimes L^{p_{2}} \otimes L^{p_{3}} \mapsto L^{q}$; we have restricted attention to the case of equal exponents $p_{j}$ primarily for simplicity. Our analysis of the rationally commensurate case actually yielded inequalities with distinct $p_{j}$, which we interpolated with trivial inequalities to make all exponents equal.

\section{References}

[1] J. Bourgain, On the dimension of Kakeya sets and related maximal inequalities, Geom. Funct. Anal. 9 (1999), 256-282.

[2] W. T. Gowers, A new proof of Szemerédi's theorem for arithmetic progressions of length four, Geom. Funct. Anal. 8 (1998), no. 3, 529-551.

[3] L. Grafakos and N. Kalton, Some remarks on multilinear maps and interpolation, preprint.

[4] G. H. Hardy and E. M. Wright, An introduction to the theory of numbers, fifth edition, the Clarendon Press, Oxford University Press, Oxford, 1985.

[5] S. Janson, On interpolation of multilinear operators, Function spaces and applications (Lund, 1986), 290-302, Lecture Notes in Math. 1302, Springer, Berlin-New York, 1988.

[6] J. P. Kahane, Trois notes sur les ensembles parfaits linéaires, Enseignement Math. 15 (1969), 185-192.

[7] N. Katz and T. Tao, Bounds on arithmetic projections, and applications to the Kakeya conjecture, Math. Res. Lett. 6 (1999), 625-630.

[8] C. Kenig and E. M Stein, Multilinear estimates and fractional integration, Math. Res. Lett. 6 (1999), 1-15.

[9] M. Lacey and C. Thiele, On Calderón's conjecture, Ann. of Math. 149 (1999), 475-496.

[10] $L^{p}$ estimates on the bilinear Hilbert transform for $2<p<\infty$, Ann. of Math. 146 (1997), 693-724.

[11] E. H. Lieb and M. Loss, Analysis, Graduate Studies in Mathematics 14 Amer. Math. Soc., Providence, 1997.

Dept. of Mathematics, University of California Berkeley, Berkeley, CA 94720.

E-mail address: mchrist@math.berkeley.edu 